\title{
An automated tool for detecting the affected area for identification of endoscopy
}

\author{
Manohar Lakkoju, Bokka Ashok kumar, Umesh Patel, Rahul Sharma , Amit Chauhan,Pavan Kumar Mishra,
}

\begin{abstract}
An automated endoscopic tool for detection of various image features from the endoscopic videos to speed up clinician process. The detection of diseased or problematic area involved in endoscopic analysis requires very enough time 7 to 8 hours to manually review the video to locate problematic area. In an attempt to automate the process, we have developed a tool which accepts the features of the problematic areas from the physician and accordingly features are detected on the image obtained from the endoscopic video. The functional modules of our tool include: Capturing image frames from a live video or already available video, Reducing redundancy involved in the images obtained such as inter-frame redundancy (Temporal) and Feature detection in images through color image segmentation and other techniques.
\end{abstract}

Index Terms - frame, endoscopy, video analysis, cancer detection

\section{INTRODUCTION}

Endoscopy is a technique in which inside of the body from the entire gut from mouth to anus is examined using a tool called endoscope. It includes the examining of the gastrointestinal tract with flexible endosco pe. It includes the insertion of an endoscope, into the human body, through a natural opening, such as the nose, mouth, or urethra. Through this tube, doctors can closely examine the insides of organs or bodily cavities. The various endoscopic procedures are gastro copy, upper gastrointestinal endoscopy, chromo endoscopy and bronchoscopes.

The endoscopic procedure involves reviewing the data captured by the endoscope which is very time consuming and tedious job. So, in order to overcome the problem and speed up the clinician process automated endoscopic procedure is introduced to automate the process without any human intervention based on automated identification of features given by the physician.

Manohar Lakkoju, Bokka Ashok kumar, Umesh Patel Department of Information Technology, NIT Raipur- 492010 Rahul Sharma

Centre for Informatics and Systems, University of

Coimbra, Portugal

Amit Chauhan

United Institute of Technology, Allahabad India

Pavan Kumar Mishra

Department of Information Technology, NIT Raipur- 492010
In this computer era image processing are extensively being used for various applications. With the increasing medical cases there is a need for automation of the computer based medical image processing and automated diagnosis of the medical images. We already have a lot of expert knowledge required for the diagnosis of commonly related medical problems and diseases. So using this expert knowledge and incorporating the same for automation of diagnosis of the disease by automatic analysis of the medical images. Automation of such process would help the clinician diagnose, prevent and use the process for research related to medical purposes. The medical image processing techniques have been majorly involved in Positron Emission Tomography, Magnetic Resonance Tomography, Diabetic Retinopathy and Computer Tomography. These areas of medical image processing have majorly used techniques of enhancement and segmentation. Endoscopic image analysis has been new in the medical image processing and only a few researchers are working on it.

The automation of image processing will improve the process of analysis and diagnosis and will majorly require working on the following areas:

Early identification of the tumors and abnormal tissues: Using the expert knowledge such as patterns of abnormal tissues and using the pattern recognition and image segmentation to identify the normal and abnormal tissue.

Acquiring measurable diagnostic data: The diagnostic data obtained can be used for serving as simulation for teaching to interns, review before surgical operations, comparing of the findings etc.

\section{LITERATURE SURVEY}

Several authors have [1-4] done tremendous effort to automated identification of cancer disease. Michal Mackiewicz et all [1] proposed colour image analysis to automatically discriminate between oesophagus, stomach, small intestine, and colon tissue in wireless capsule endoscopy.WCE uses "pill-cam" technology to recover colour video imagery from the gastrointestinal tract.

In 2007 American Society for Gastrointestinal Endoscopy provided review of existing, new or emerging endoscopic technologies that have an impact on the practice of gastrointestinal endoscopy. Veronese,E. Et al [4] proposed Hybrid patch-based and image-wide classification of confocal laser endomicroscopy images in Barrett's esophagus surveillance. They also focus on a computer-based method for 
the automatic classification of gastric metaplasia (GM), intestinal metaplasia (IM) and neoplasia (NPL) on the basis of appearance features of confocal images. Jitendra Malik et al [5] proposed an algorithm for partitioning grayscale images into disjoint regions of coherent brightness and texture. Jianbo Shi and Jitendra Malik, Member, IEEE proposed a novel approach for solving the perceptual grouping problem in vision. . Farhan Riaz , et al [8] proposed Impact of Visual Features on the Segmentation of Gastroenterology Images Using Normalized Cuts.

\section{WORKING ARCHITECTURE}

We have described the architecture of an automated tool in figure 2. This tool takes the input video from the bio medical device. The size of video is too large so it needs to reduce the video frame because most of frames are similar to previous fame. The detail description of this tool is given below.

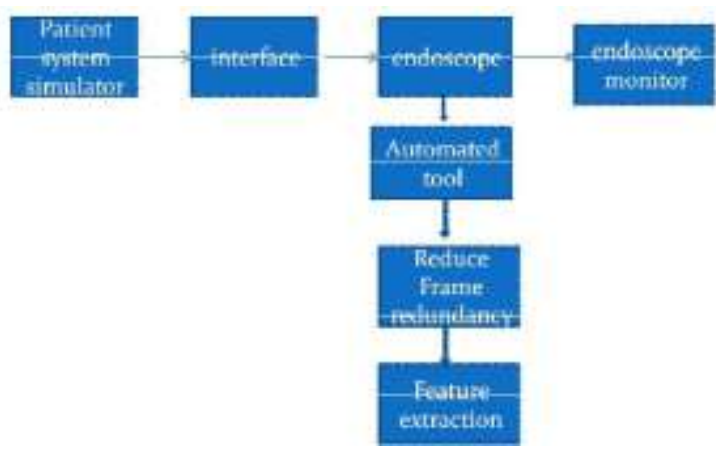

Figure 1 Architecture of automated tool

Patient System Simulator: Patient Simulator is a realistic, hands-on simulator of the anesthetized or critically ill patient. A "hands-on" simulator is one in which the clinical environment and the patient are represented as real physical objects. A specially instrumented patient mannequin stands in for the patient, and real clinical equipment is used to make up the work environment.

Interface: The interface or the device which exists between the patient and the endoscopic device.

Endoscope: An endoscope is a medical device consisting of a long, thin, flexible (or rigid) tube which has a light and a video camera. Images of the inside of the patient's body can be seen on a screen.

Endoscope Monitor: Equipment for patients undergoing procedures includes pulse oximetry, single-lead continuous electrocardiographic (Ecg) monitoring and automated blood pressure monitoring.

Automated Tool: An application tool developed for automated endoscopic video analysis such that any new algorithm can be added and be implemented for the image processing. The application serves the purpose of diagnosis

Frame Redundancy: We remove the temporal redundancy by comparing the consecutive images and obtaining the difference between the images. if the difference is above the threshold then both the frames are different and if the difference is below the threshold then one of the frame can be removed since it is redundant.

Feature Extraction: This phase involves feature detections such as red spot and color image segmentation to recognize the wounded portion in the frame that is selected in order to find the resultant of the diagnosis we have to feature out the wounded portion separately from the image such that we can know the mildness of the disease. Here we used the color image segmentation technique for recognizing the red spot or the wounded portion which has more red intensity as compared to other parts

\section{WORKING MODEL}

We have developed an automated tool which supports endoscopic process. Endoscopic tool is created by using the qt creator which is a cross-platform application framework that is widely used for developing application software with a graphical user interface (GUI).This tool feature's in recording a video from the web cam and saving the required frame for analysis or browse the required frame from the system that should be analyzed. we have incorporated some fundamental algorithms like histogram equalization, edge detection etc in form of a drop down box such that the algorithm that was selected can be applied on the selected image.

We used Qt which is a cross-platform application framework that is widely used for developing application software with a graphical user interface (GUI). To develop the tool we used openCV is an Image Processing library created by Intel.

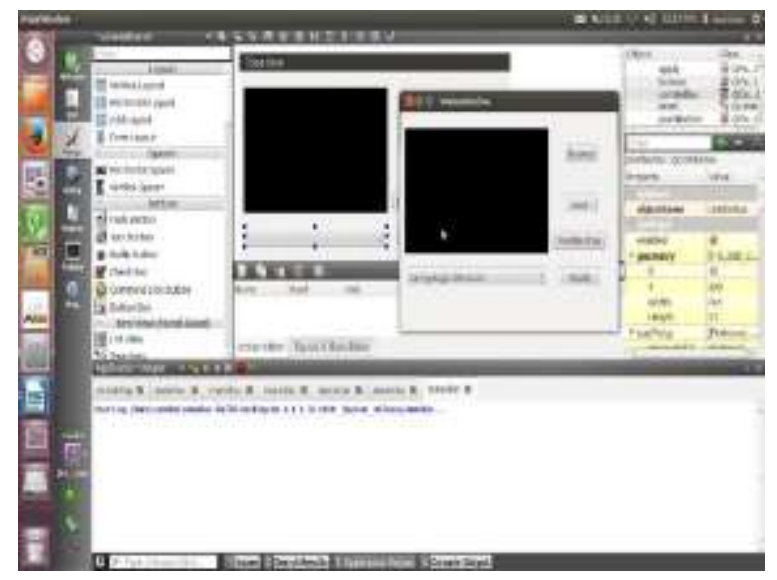

The next phase in the project involves the frame redundancy. The video obtained from the endoscope which has capacity of storing a video of about 7-8 hours has about 50,000 frames which consist of redundant frames. It is important to get set of distinct frames for applying frames for feature detection. 
We know that there are about 24 frames captured per second through an endoscope video. So the frames involved might have temporal redundancy i.e. containing the same information in consecutive images.. In order to remove those images with same features or temporal redundancy. We remove the temporal redundancy by comparing the consecutive images and obtaining the difference between the images . if the difference is above the threshold then both the frames are different and if the difference is below the threshold then one of the frame can be removed since it is redundant

Since each image is considered as a matrix of consecutive rows and columns as their pixel intensities, So the images can be subtracted to get the differences between the images. We convert all the images into grayscale. In a grayscale the image consists of values between 0-255 and when the two images are subtracted the resultant value will also be between 0-255.

Noise will also effect the images and sometimes due to the noise factor even when the images are same they don't get a null value as output on performing difference so, here we will take an assumption that the effect of noise is upto a range of 5 in the output hence we consider a threshold value ' $d$ ' which acts as a scaling factor whether the images are the same or they are different

Method :

- convert the images into grayscale

- select the two consecutive images and apply difference between the two images

- if the difference between the images is less than or equal to 5 then both the images are the same and one image can be discarded

- else if the difference between the images is greater than 5 then both the images are different and we cnnot discard any of them

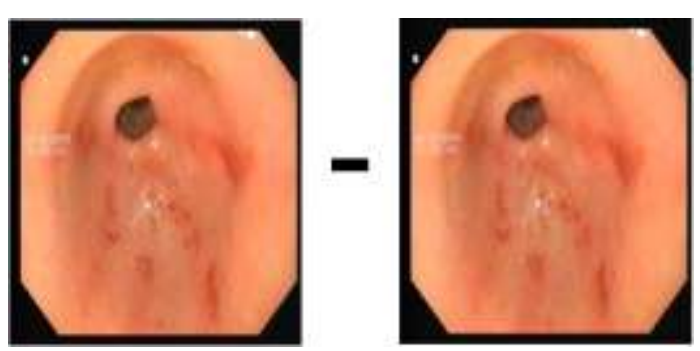

\section{Better approach :}

Considering all the consecutive frames and comparing them is a complex procedure so to have an efficient comparison we suggested a better technique in our work by using the fact that 24 frames are extracted per second from a video through the endoscopic device

Here we are taking an assumption that the endoscopic device is moving at a rate of $1 \mathrm{~cm} / \mathrm{sec}$. Hence 24 frames are captured in one second covering a distance of $1 \mathrm{~cm}$. hence instead of comparing each frame one by one its fruitful to compare the first frame and the $24^{\text {th }}$ frame and if they are same ie, difference less than threshold then we can remove all the frames from 1 to 23 since they are all the same now we have to consider the $24^{\text {th }}$ frame and the $48^{\text {th }}$ frame ie, nth frame and $(n+24)^{\text {th }}$ frame and continue the process further. Else if the difference between the $1^{\text {st }}$ frame and the $24^{\text {th }}$ frame is greater than the threshold then we have to use binary search type of technique here ie, comparing the $1^{\text {st }}$ and the $12^{\text {th }}$ frames and so on . thus through this method the complexity of $\mathrm{O}(\mathrm{n} 2)$ is converted into $\mathrm{O}(\log 2 \mathrm{n})$

This method thus reduces unnecessary comparisions of the redundant frames and also helps in removing some of the redundant frames without even performing the comparisons by taking the advantage of the sorted frames in an endoscopic video analysis .

\section{FEATURE EXTRACTION}

The third phase involves feature detections such as red spot and colour image segmentation to recognize the wounded portion in the frame that is selected . in order to find the resultant of the diagnosis we have to feature out the wounded portion separately from the image such that we can know the mildness of the disease. Here we used the color image segmentation technique for recognizing the red spot or the wounded portion which has more red intensity as compared to other parts.

Red Spot detection : Delineate each individual spot area, outputting a list of

spot centres, intensities and geometric features. The spot detection operations pipeline in

most cases is to detect the centres of as many spots as possible and segment the gel into regions, each containing one of these spots.

The phases in red spot detection are:

Conversion of the RGB image into Gray scale : The image which is in rgb mode is to be converted into gray scale mode which have values ranging from 0 to 255., where 0 denotes black colour and 255 denotes white colour. By converting the image into gray scale mode we can easily able to identify or segment the colors based on the gray scale values of the colours.
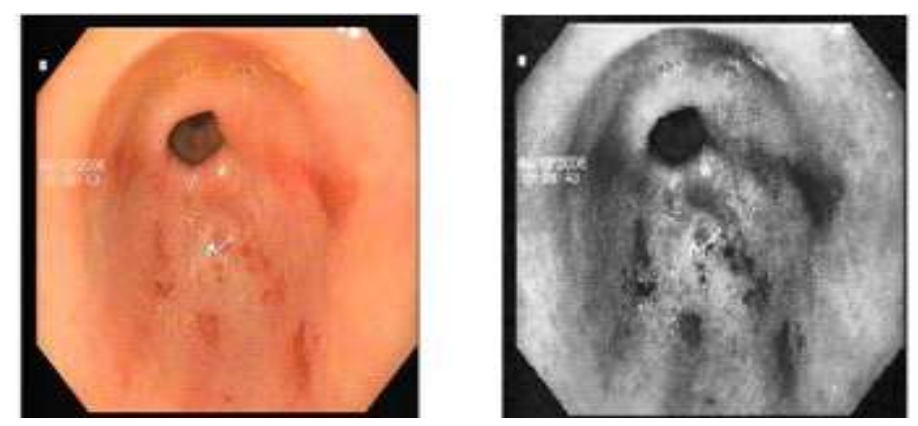

Figure 2 Conversion of an RGB image to Gray scale

Apply filter : Apply any filters like guassian filter etc on the image such that the image is smoothened.the darks spots or the awkward portion on the image 
Proc. of the Intl. Conf. on Advances in Computing, Control and Networking - ACCN 2015.

Copyright (c) Institute of Research Engineers and Doctors, USA .All rights reserved.

ISBN: 978-1-63248-038-5 doi: 10.15224/ 978-1-63248-038-5-04

which is considerd as noise might be accumulated at a particular portion on the image causing disturbances in the process of image segmentation . hence, we use a smoothening filter such as a guassian filter which helps in reducing the noise.

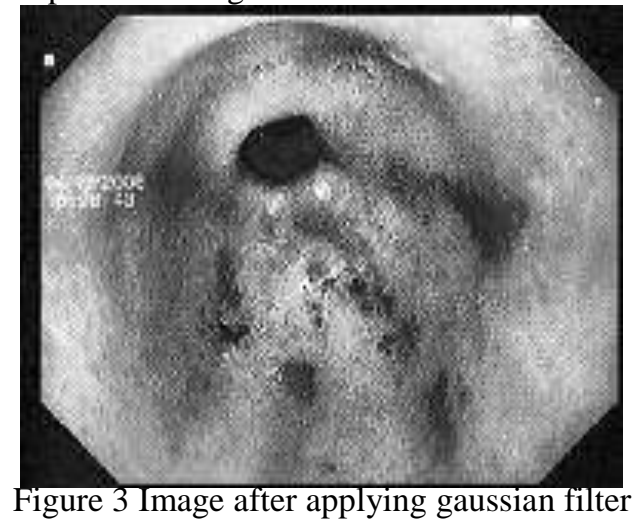

Now apply adaptive histogram : The next step is to apply adaptive histogram on the image such that the color of the image gets enhanced. Adaptive histogram equalization (AHE) is a computer image processing technique used to improve contrast in images. It differs from ordinary histogram equalization in the respect that the adaptive method computes several histograms, each corresponding to a distinct section of the image, and uses them to redistribute the lightness values of the image. It is therefore suitable for improving the local contrast of an image and bringing out more detail.

Thresholding : Now, we use the thresholding technique in order to recognize the brighter part separately in the image. This can be done by setting a threshold value of intensity. The pixels above the threshold will be considered and the rest of the pixels are treated as null. Thus, separating the brighter region from the darker

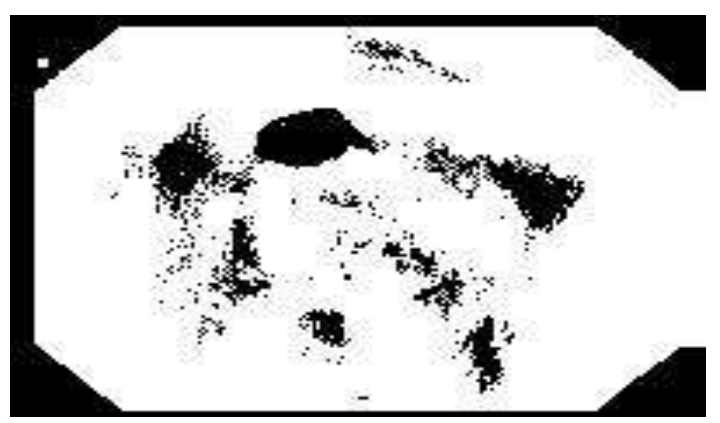

Figure 4 Image after applying thresholding technique

Mapping : Now, map the darker region with the red colour. Set the parameters of the 3 channel mode that is the RGB mode in such a way that the darker portion corresponds with the red region. Thus the portions of red spots ie, the wounds are clearly identified by this method .

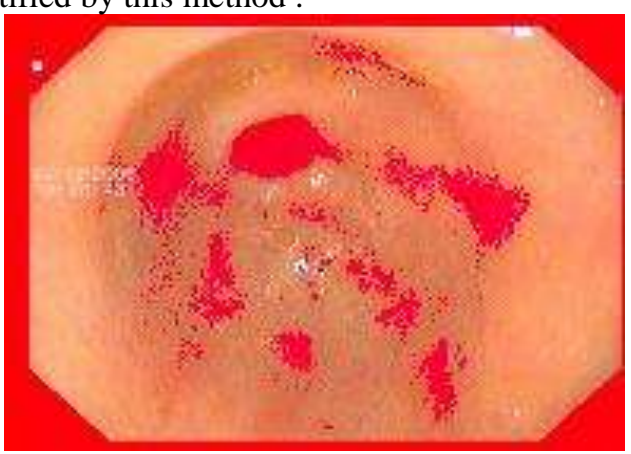

Figure 5 Image after mapping the darker region to red colour

Cancerous cells detection : Most of the endoscopic diagnosis is used in detection of cancerous cells . In generally cancer is either determined through the tumors, bulb like outer projections or through the cancerous cells over growth . Here we are extracting diagnosis of the cancer based on the cancerous cells ie, the ite external growth on the regular organs .

Phases in cancerous cells detection :

Conversion of the RGB image into Gray scale : The image which is in rgb mode is to be converted into gray scale mode which have values ranging from 0 to 255., where 0 denotes black colour and 255 denotes white colour. By converting the image into gray scale mode we can easily able to identify or segment the colors based on the gray scale values of the colours

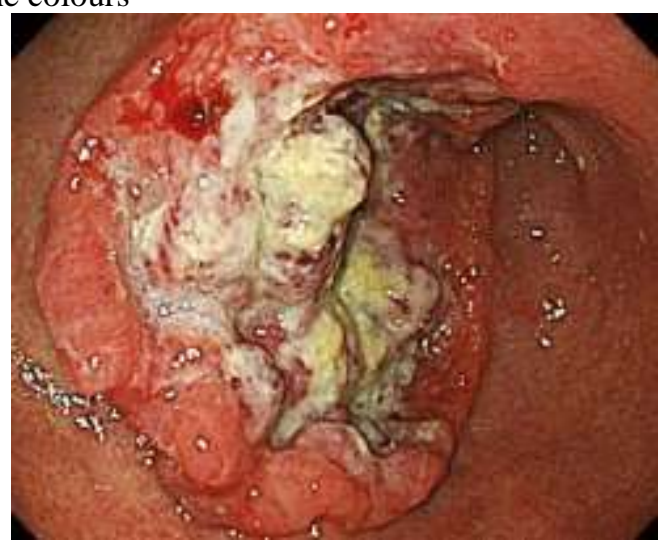

Figure 6 cancerous image

Thresholding : Now, we use the thresholding technique in order to recognize the brighter part separately in the image. This can be done by setting a threshold value of intensity. The pixels above the threshold will be considered and the rest of the pixels are treated as null. Thus, separating the brighter 
Proc. of the Intl. Conf. on Advances in Computing, Control and Networking - ACCN 2015.

Copyright (C) Institute of Research Engineers and Doctors, USA .All rights reserved.

ISBN: 978-1-63248-038-5 doi: 10.15224/ 978-1-63248-038-5-04

region from the darker

Note : here we donot consider adaptive histogram because we need to separate the whiter region

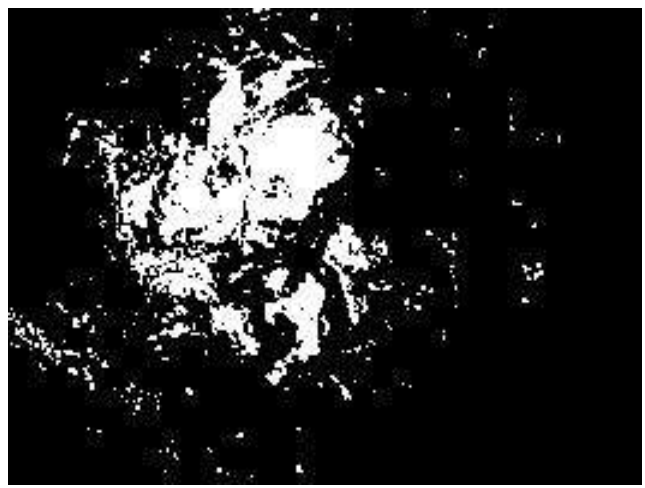

Figure 7 Image with brighter part separate after Thresholding technique

Mapping : Now, map the darker region with the red colour. Set the parameters of the 3 channel mode that is the RGB mode in such a way that the darker portion corresponds with the Green or the blue channel (green preferably) .Thus the portions of white spots ie, the infected area is clearly identified by this method

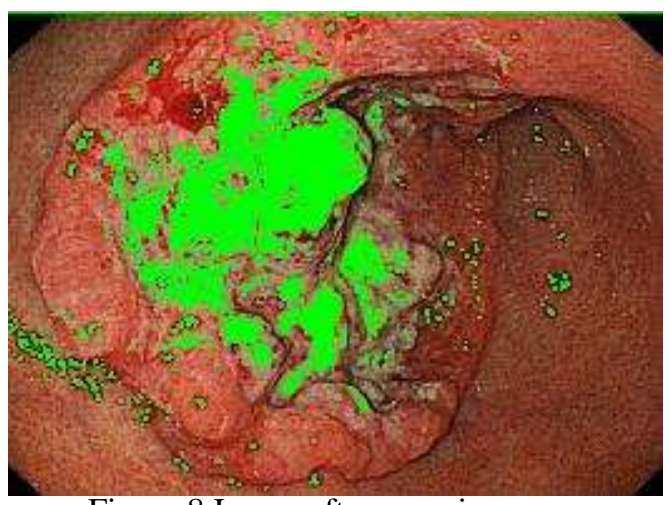

Figure 8 Image after mapping

\section{CONCLUSION}

The tool developed for automated endoscopy can be used for medical purposes as well as research purpose as we have provided the tool with scalability providing it with capability to incorporate new algorithms. Our tool has been developed on a cross platform framework so it can work on any platform or machine configuration provided the QT framework for that platform is present. While used for medical purposes it is time saving and efficient for reviewing the endoscopic videos. The tool is time saving as feature extraction is applied on the distinct frame after removing redundant frames. The clinician reviewing process may involve missing of details from the video being analysed but automated endoscopic analysis ensures covering all the details of the video being analysed.

\section{VII.REFERENCES}

[1] G. Eason, B. Noble, and I. N. Sneddon, "On certain integrals of Lipschitz-Hankel type involving products of Bessel functions," Phil. Trans. Roy. Soc. London, vol. A247, pp. 529-551, April 1955. (references)

[2] J. Clerk Maxwell, A Treatise on Electricity and Magnetism, 3rd ed., vol. 2. Oxford: Clarendon, 1892, pp.68-73.

[3] I. S. Jacobs and C. P. Bean, "Fine particles, thin films and exchange anisotropy," in Magnetism, vol. III, G. T. Rado and H. Suhl, Eds. New York: Academic, 1963, pp. 271350.

[4] Veronese, E.; Grisan, E.; Diamantis, G.; Battaglia, G.; Crosta, C.; Trovato, C., "Hybrid patch-based and image-wide classification of confocal laser endomicroscopy images in Barrett's esophagus surveillance," Biomedical Imaging (ISBI), 2013 IEEE 10th International Symposium on , vol., no., pp.362,365, 7-11 April 2013

[5] Jitendra Malik, Serge Belongie, Thomas Leung, Jianbo Shi "Contour and Texture Analysis for Image Segmentation" International Journal of Computer Vision, 2001.

[6] Y. Yorozu, M. Hirano, K. Oka, and Y. Tagawa, "Electron spectroscopy studies on magneto-optical media and plastic substrate interface," IEEE Transl. J. Magn. Japan, vol. 2, pp. 740-741, August 1987 [Digests 9th Annual Conf. Magnetics Japan, p. 301, 1982].

[7] M. Young, The Technical Writer's Handbook. Mill Valley, CA: University Science, 1989.

[8] Riaz, F.; Silva, F.B.; Ribeiro, M.D.; Coimbra, M.T., "Impact of Visual Features on the Segmentation of Gastroenterology Images Using Normalized Cuts," Biomedical Engineering, IEEE Transactions on, vol.60,
no.5,
pp.1191,1201,
May
2013 